\title{
En busca del nicho apropiado. Lógicas de acción de las escuelas y el surgimiento de un escenario institucional diversificado
}

\author{
Verónica Gottau \\ CEPE- Di Tella, Escuela de Gobierno. Argentina \\ https://orcid.org/0000-0002-7991-6761 \\ vegottau@gmail.com \\ Liliana Mayer \\ Universidad Nacional de Misiones / Consejo Nacional de Investigaciones en Ciencia \\ y Técnica (CONICET), Argentina \\ https://orcid.org/0000-0002-7364-4048 \\ lzmayer@gmail.com
}

Recepción: 25-05-2020

Aceptación: 09-11-2020

Publicación: 17-02-2021

\section{Resumen}

Este trabajo parte de la hipótesis de que las escuelas primarias y los colegios secundarios despliegan diferentes estrategias para determinar su perfil y proyecto institucional para definir los públicos que desean albergar. A partir del marco conceptual de las lógicas de acción propuesto por Ball y Maroy (2009) y Van Zanten (2009), realizamos un análisis de las lógicas de acción que implementan las 14 escuelas no subvencionadas de la Ciudad de Buenos Aires ubicadas en la Comuna 13 para determinar su posicionamiento dentro del espectro educativo. Entre los principales resultados, destacamos cuatro tipos de lógicas de acción predominantes, aunque no excluyentes, que tienden a determinar el perfil de los alumnos de la escuela y su posicionamiento social: (a) lógicas customizadas — escuelas a la carta-; (b) lógicas de ensamblaje — escuelas de largo plazo—; (c) lógicas vanguardistas — escuelas de punta- y (d) lógicas globales — escuelas que se internacionalizan. Concluimos que las escuelas no son actores pasivos o meros receptores de las elecciones de los padres. A través de sus lógicas de acción y estrategias de posicionamiento, intentan proyectar una imagen en línea con el proyecto institucional y el perfil de los alumnos o, más precisamente, el perfil de los padres que desean capturar. El análisis hasta aquí expuesto permite comprender los procesos de diversificación de instituciones dentro de circuitos homologables entre sí.

Palabras clave: diversificación escolar privada; organización escolar; educación urbanalógicas de acción; Buenos Aires 
Abstract. Finding the right niche. Schools logics of action and the emergence of diversified institutional landscape

This work relies on the hypothesis that primary and secondary schools develop strategies to determine the social position of the school in the local area and the profile of the enrolment they try to capture. We draw in the conceptual framework proposed by Ball y Maroy (2009) y Van Zanten (2009) to analyse the logics of action implemented by 14 non-subsidized schools located in the borough 'Comuna 13' in the City of Buenos Aires. The results show that there are four mainstream though not exclusive logics of action: (a) Customized logics of actions: school a la carte; (b) Assembly logics: in the long run schools; (c) Avant-garde logics: cutting edge schools and (d) Worldwide Logics: schools that go global. We conclude that schools are not passive receivers of students or parents' schools choice. They develop strategies and implement different logics of action to position themselves in the local area and determine school enrolment, or more specifically, the profile of the families they aim at. This work sheds some light on the processes of diversification of schools with similar characteristics located in the same neighbourhood.

Keywords: private school diversification; school organization; urban education; logics of action; Buenos Aires

\begin{aligned} & \multicolumn{2}{c}{ Sumario } \\ & 1. Introducción $\begin{array}{l}\text { 4. Escuelas y posicionamiento social } \\ \text { en cuasimonopolios educativos }\end{array} \\ &$ 2. Consideraciones conceptuales y \\ & metodológicas Referencias bibliográficas \\ & $\begin{aligned} \text { 3. Sobre las escuelas } \\ \text { y sus lógicas de acción }\end{aligned}$ Anexo \end{aligned}

\section{Introducción}

Desde la perspectiva de análisis de la organización y gestión de los sistemas educativos nacionales, las reformas introducidas en las décadas de 1980 y 1990 produjeron una serie de cambios estructurales que tendieron a conformar un nuevo paradigma educativo. Si bien en términos generales los sistemas educativos se reorientaron hacia estrategias ligadas a las lógicas del mercado, como podría ser el caso de los Estados Unidos, Nueva Zelanda o Inglaterra, Argentina pasó por un proceso diferente, en el que tales modificaciones no ocurrieron, al menos no con la misma intensidad que en sus países vecinos (Zancajo, 2018; Beech y Barrenechea, 2011). En términos generales, la historia del sistema argentino está fuertemente orientada hacia lo estatal, ${ }^{1}$ lo que da

1. Esto es así desde los comienzos del sistema educativo argentino, regulado por la Ley 1420, de 1884, donde el Estado asumía una función fundamental en la formación en el nivel primaria. Aun con los cambios propios del desarrollo del Estado nación, este ethos se mantuvo, con las modificaciones y nuevas legislaciones educativas, primero en 1993 con la Ley Federal de Educación y principalmente con la Ley de Educación Nacional actualmente vigente, sancionada en el año 2006. 
como resultado que muchos de los procesos insertados en otras latitudes fueran bloqueados y rechazados particularmente en el subsistema estatal (Narodowski et al., 2017). Por su parte, el sector de escuelas de gestión privada mostró una posición «intermedia», en la que muchas de sus instituciones adoptaron narrativas de las reformas e incorporaron algunos componentes - muchas veces por default - orientados a lógicas mercantiles (Mayer, 2020). Esto se ha visto reflejado, en particular, respecto a la configuración de su perfil educativo y proyecto pedagógico, en el marco de un subsistema que adquiría niveles de autonomía cada vez mayores, principalmente en aquellas instituciones que no reciben financiamiento estatal (Morduchowicz, 2005).

En este marco, retomamos las ideas de Delvaux y Van Zanten (2006), quienes destacan que las escuelas reciben una doble influencia: externa - por normas, políticas, directivas y recomendaciones - e interna - por la actividad de los directores, maestros, padres y alumnos, que se ven afectados a su vez por las configuraciones locales. De esta manera, las escuelas son construidas por el sistema educativo al tiempo que lo determinan. ${ }^{2}$ Un análisis a partir de la micropolítica (Ball, 1989) permite una mirada acerca de las escuelas como espacios de puja de poder, donde los actores luchan por el control y donde se cristalizan distintos objetivos e intereses que están ideológica y valorativamente orientados. Las formas concretas y singulares en que se desarrollan estas micropolíticas construyen la macropolítica. En este contexto, nuestro análisis parte de la hipótesis de que las instituciones despliegan diferentes estrategias, conscientes o no, para determinar su perfil y proyecto institucional, y con estos aspectos definir los públicos que desean albergar. En vista de lo expuesto, este trabajo se propone realizar un análisis de las lógicas de acción que implementan las escuelas de la Comuna 13 sin subvención (barrios de Núñez y Belgrano), para determinar su posicionamiento dentro del espectro educativo. Hay que recordar que esto es posible, entro otras causas, porque estas escuelas gozan de mayores márgenes de autonomía para la toma de decisiones institucionales que sus pares estatales o privadas subvencionadas.

\section{Consideraciones conceptuales y metodológicas}

El marco conceptual propuesto por Ball y Maroy (2009) y Van Zanten (2009) permite comprender las diversas actividades desarrolladas por las escuelas en

2. El artículo se nutre de múltiples perspectivas, y se adapta a los formatos, límites y extensiones generales de la convocatoria. En este punto, un eje central corresponde a los usos del lenguaje inclusivo en la redacción del mismo. Al igual que otros aspectos de la vida social, el lenguaje es dinámico y sujeto a transformaciones; se entiende que el mismo no es neutral y su uso tiene consecuencias ideológicas. Sin embargo, teniendo en cuenta que el uso del lenguaje inclusivo aún no ha sido académicamente resuelto y, dada la extensión máxima establecida para la propuesta, hemos decidido no utilizarlo y mantener los genéricos, con la excepción de los casos en que sea necesario indicar lo contrario. La referencia a «los padres», «los directores», «los docentes» y «los alumnos» y demás sustantivos engloba la pluralidad de géneros posibles y la diversidad de casos hacia dentro de cada uno de ellos. La falta de acuerdos respecto a su uso nos llevó a declinar su inclusión y especificar la condición genérica cuando sea necesario. 
su determinación de perfil y proyecto educativo. En este marco, los autores destacan que, a diferencia del concepto de estrategia, el cual supone cierta racionalidad y la persecución de un objetivo determinado, el término «lógicas de acción» no supone racionalidad instrumental, sino acciones que pueden responder a diversos criterios. A su vez, las lógicas de acción se refieren a las orientaciones predominantes asociadas a las conductas institucionales en diferentes esferas de acción, toma de decisiones, rutinas o decisiones prácticas, y son reconstruidas ex post facto por el observador (Maroy y Van Zanten, 2009: 72). De esta manera, el conjunto de lógicas implementadas por las instituciones educativas estará condicionado por factores internos y externos: los primeros se refieren a la narrativa escolar, el reclutamiento de alumnos, las diversas opciones pedagógicas, la publicidad, el seguimiento y la atención de necesidades especiales y regulaciones acerca de la conducta; mientras que los factores externos remiten a la posición de la escuela en el espacio local de interdependencia y a las políticas y regulaciones y la percepción de estas por parte de los padres.

Como mencionamos líneas arriba, las lógicas de acción implementadas por las escuelas no son necesariamente el resultado de un discurso estructurado, sino que corresponden más bien al conjunto de actividades desarrolladas por la escuela y sus diversas formas de comunicación institucional (Maroy, 2004). Por lo tanto, estas lógicas deben ser reconstruidas ex post facto y, lejos de ser excluyentes, pueden presentarse como mutuamente complementarias. Vale decir que, si bien algunas lógicas de acción son excluyentes, como por ejemplo ofrecer los tres niveles de educación obligatoria o no, otras pueden resultar accesorias a otras para conformar el proyecto. Esta reconstrucción analítica supone entonces la posibilidad de encontrar diferentes lógicas convivientes dentro de una misma institución. A diferencia de las tipologías, las lógicas de acción son entendidas como herramientas heurísticas que permiten organizar el comportamiento institucional en función de los repertorios y narrativas predominantes en las instituciones, donde pueden prevalecer unas sobre otras o conjugarse de manera recíproca e interdependiente.

En vista de lo expuesto, estudiar las instituciones permite a su vez dar cuenta del sistema educativo y de las desigualdades sociales y educativas, así como de los procesos de fragmentación, que también se construyen y producen a partir de las prácticas de estos sectores (Tiramonti, 2004; 2008), que podemos denominar de "élite» (Bourdieu, 2016). Mills (1956) aseveraba que las instituciones que albergan estos públicos son la agencia más importante para trasmitir las tradiciones de los sectores sociales acomodados y las regulaciones para acceder a las nuevas riquezas. Estas instituciones suelen pertenecer al subsistema de educación privado, usualmente relegado en las investigaciones en nuestro continente, pese a ser el espacio geográfico donde de diversas maneras y con diferentes impulsos más ha crecido la matrícula en los últimos años (Verger et al., 2017). Bourdieu (2016) sostiene que en las sociedades modernas de diferenciación creciente corresponde referirse a clases dominantes en plural, debido a la constitución de diversos campos de poder con capitales propios, donde además se distingue la tenencia de capital económico y la de 
capital cultural. Las sociedades modernas valoran de manera creciente la cultura, al tiempo que adquiere ciertos niveles de autonomía de la posesión - $\mathrm{O}$ acumulación - de capital económico, lo que establece distinciones estéticas y semánticas. De allí que las élites —o, más precisamente para los objetivos de este trabajo, las escuelas consideradas de élite- no pueden entenderse como un sector homogéneo, sino con intereses y prácticas divergentes que tienden a diferenciarse dentro de un espectro determinado.

Para el análisis de las escuelas, nos valdremos del trabajo de campo comenzado en el año 2018, en el que se entrevistaron ${ }^{3}$ a agentes educativos - autoridades y docentes - de escuelas de la Comuna 13 de la Ciudad de Buenos Aires. ${ }^{4}$ En muchos casos, las entrevistas estuvieron acompañadas por observaciones de clases, trabajo de archivo en las instituciones y análisis de documentos programáticos y de las páginas web de 14 instituciones. Para nuestro artículo, seleccionamos las escuelas primarias y secundarias de la Comuna 13 que no reciben ningún tipo de financiamiento por parte del Estado y por lo tanto no tienen tope arancelario. ${ }^{5}$

Así, quedan fuera de esta selección las escuelas privadas confesionales católicas tanto de nivel primario como secundario de la Comuna 13, dado que son escuelas que reciben subvenciones por parte del Estado. Si bien las escuelas privadas son percibidas como un único universo, desde la perspectiva de análisis de la política educativa existe una gran heterogeneidad en su interior respecto al porcentaje de subvención estatal que reciben, principalmente por dos cuestiones en particular: (a) a mayor porcentaje de subvención, menor el arancel, y (b) la gran mayoría de las escuelas privadas que reciben subvenciones son católicas, y las nuevas escuelas privadas laicas en su gran mayoría no reciben subvenciones.

Para tal fin, se analizaron siete escuelas primarias sin continuidad en el nivel secundario y siete escuelas secundarias sin financiamiento estatal, de las cuales cuatro ofrecen los tres niveles educativos y tres solo ofrecen nivel secundario. Las especificidades de cada escuela se encuentran en el Anexo. Para el análisis

3. En todos los casos entrevistados, sus repertorios fueron abordados comprensivamente, es decir, teniendo en cuenta los contextos específicos dentro de los que los mismos se desarrollan. Desde nuestra perspectiva, todo actor social, al narrar una situación determinada, la produce. Con relación a nuestro diseño metodológico, coincidimos con Saltalamacchia cuando señala que el individuo es «un lugar de "anudamiento" de un conjunto determinado de relaciones sociales» (1992: 38). La entrevista debe ser entendida como el marco de una elaboración teórica conjunta en la que no necesariamente surgirán acuerdos totales o aprovechamientos semejantes, pero sí interpretaciones que no existían antes de la relación (Saltalamacchia, 1992).

4. El subsistema privado de educación en Argentina estuvo desde sus inicios conformado por escuelas comunitarias de diversos orígenes migratorios que aspiraban a integrarse socialmente, a la par que conservar sus tradiciones, acervos y lengua materna, y escuelas confesionales católicas. Estas últimas tuvieron y continúan teniendo financiamiento estatal, que en algunos casos puede cubrir hasta el 100 por ciento de la cuota mensual. Para ampliar este tema, ver Torres, G. (2014).

5. La Ley 13047 estipula un porcentaje de subvención por parte del Estado nación a las escuelas condicionado a requerimientos y pautas específicas que determinan a su vez el precio del arancel escolar. 
de las entrevistas, se utilizó la aplicación Atlas ti a partir de un modelo inductivo-deductivo. La primera codificación se realizó según los criterios conceptuales extraídos del marco teórico, y las siguientes codificaciones se hicieron por capas con categorías nativas. Considerando que las entrevistas se sustentan en las narrativas de los entrevistados, en todo momento recurrimos a la repregunta como modo de reinterpretar el sentido enunciado y construido. Intentamos ir más allá de la premisa de la que partía la narrativa, ya que entendemos que toda argumentación constituye un constructo social.

Esto proporciona un recorte metodológico por un lado y teórico por el otro, ya que se trata de instituciones que, de manera independiente a sus proyectos institucionales y a sus diferenciaciones entre sí, se homologan por albergar a los sectores socioeconómicos aventajados de la ciudad y en muchos casos del área metropolitana, conocidas como «escuelas de élite». Quedan fuera de nuestro análisis instituciones religiosas de la Iglesia católica, al recibir financiamiento estatal, aun cuando alberguen públicos homologables.

La Comuna 13 presenta menores niveles de vulnerabilidad socioeconómica y comprende la mayor cantidad de escuelas de gestión privada en la jurisdicción. Esta circunstancia nos permite observar las diferentes lógicas de acción que despliegan las instituciones para delimitarse y diferenciarse. ${ }^{6}$

\section{Sobre las escuelas y sus lógicas de acción}

\subsection{Lógicas customizadas: escuelas a la carta}

Una de las características en términos escolares de la Comuna 13 es la gran cantidad de escuelas primarias privadas laicas sin continuidad en el nivel secundario. La particularidad de ofrecer un solo nivel conforma un universo particular de instituciones que suponen lógicas de acción específicas, a diferencia de otras instituciones que, como veremos a continuación, ofrecen todos los niveles de enseñanza obligatoria. No es casual que estas instituciones ofrezcan solo el nivel primario, sino más bien intencional, ya que no hay interés institucional de ofrecer continuidad. Se trata de escuelas relativamente nuevas que surgieron en la década de 1990 y que funcionan en edificios relativamente pequeños comparados con sus pares estatales.

El análisis de la discursividad institucional sugiere que estas escuelas buscan en general fomentar el desarrollo de la subjetividad, la personalidad y los recursos personales. Esto no implica que dejen de lado criterios de equidad, sino que aspiran a un desarrollo de lo individual por encima de lo colectivo. En palabras de una de las directoras de las escuelas primarias: «En general, nosotros tenemos un equipo psicopedagógico que trabaja muy bien, porque trabajamos sobre las características del chico. Y es la escuela la que se adapta al chico y no al revés $[\ldots] »$ (directora de la escuela $\mathrm{F})$.

6. La participación estatal de la matrícula del nivel primario para la Comuna 13 es del 30,7 $\%$, aunque asciende al $70,1 \%$ en la comuna 4 . La participación estatal de la matrícula del nivel secundario es del 67,6 \% y el 15,1\% para las comunas 4 y 13 , respectivamente. 
En todo momento, las directoras entrevistadas resaltaban la importancia de la inclusión social, del cuidado y del manejo de los problemas sociales. La directora de la escuela $\mathrm{C}$ agregaba: «Son familias de clase media, media-alta. Tienen una mirada de mucho cuidado y están atentos a lo que pasa acá». Por su parte, y en similar línea de análisis, el director de la escuela B afirmaba:

A la mayoría de los padres que eligen este colegio les interesa el desarrollo del chico, les interesa su inserción en la sociedad, tanto para ellos como para nosotros; hay una sociedad en el medio, hay que prepararlos para esto. No les interesa ser parte de una élite, aunque sí lo es en cierta manera por el tipo de cuota, pero no quieren fomentar que vivan en una burbuja.

Por otro lado, el énfasis puesto en el desarrollo y el tratamiento individual, como se puede observar en sus páginas web, también delimita un sector especifico de la matrícula. Así, por ejemplo, algunas de las frases enunciadas por los entrevistados son: «Estimular al máximo el potencial de cada niño, sin sobreexigir sus etapas de maduración» o «Un espacio [...] con respeto por los tiempos y modalidades individuales [...] donde los chicos se sientan seguros y puedan disfrutar a pleno» (directora de la escuela G).

Las familias son un aspecto fundamental para la escuela que plantea una vinculación estrecha, donde los padres son vistos como parte esencial de la institución, y en todo momento buscan su participación y colaboración. Esta mirada respecto a las familias configura un esquema de escuela tan participativa como demandante, que se ancla en prerrequisitos previos de la familia, en lo que refiere a su disponibilidad temporal, por un lado, pero también en términos de capital cultural (Bourdieu, 1987). «Nosotros les preguntamos en la entrevista o cuando vienen a conocer el colegio: ¿ustedes tienen tiempo? Porque en esta escuela trabajamos mucho con los padres», afirmaba la directora de la escuela $A$.

En línea con lo sostenido líneas arriba, la directora de la escuela E argumentaba:

La población de padres de esta escuela es muy particular. Esta escuela se elige, no es porque está en el barrio y listo. Es más, hay familias de lejos que se mudan para venir acá. Son poblaciones con mucho criterio de realidad, participan mucho socialmente, políticamente formados, mucha conciencia social.

La intención de la directora fue posicionar a la escuela como una institución que se elige no por la comodidad de la cercanía, sino porque es "particular» y «especial». Desde su perspectiva de análisis respecto a los padres, es una población con sentido de realidad, que no viven en una burbuja y que procuran que sus hijos no crezcan en ese ambiente. Resulta de interés resaltar el hecho de que ni los directores ni los padres, al menos desde la perspectiva de análisis de la directora, se autoperciben como «élite» $y$, sin embargo, este grupo de escuelas primarias tiene los aranceles más altos de toda la Ciudad de Buenos Aires. En palabras de la directora de la escuela $\mathrm{G}$, «hay una parte de la población que dice 
"yo traigo a mi hijo a esta escuela y después [para el nivel secundario] lo llevo a una escuela de gestión estatal". En general, va variando cuál es esa escuela».

Es válido aclarar que estas escuelas estatales que menciona son consideradas de élite principalmente por al menos tres motivos: a) en su mayoría, se trata de instituciones dependientes de la Universidad de Buenos Aires, b) requieren de un riguroso examen de ingreso cuya preparación es particular y privada, con un costo mensual similar al arancel de una escuela primaria sin subvención, y c) su nivel de exigencia por lo general excluye a alumnos que no asistieron a buenas escuelas primarias. En vista de lo expuesto, podríamos argumentar que, junto con lo "particular» $\mathrm{y}$ «especial» de estas instituciones, se suman las narrativas respecto a su "calidad" y "excelencia académica", por ser instituciones que logran hacer ingresar a sus alumnos en tales instituciones: «Bueno, la escuela tiene algo fundamental que es el método de trabajo. Trabajamos con el método científico, o sea, que averigüen, investiguen, pregunten» (directora de la escuela F).

En síntesis, se trata de escuelas que, sin renunciar a la "excelencia académica", proponen un proyecto educativo que combina esquemas participativos familiares, contención y personalización, en particular a los ritmos de aprendizaje; son escuelas dialógicas, donde la voz de los padres tiene su lugar. En este sentido, este conjunto de escuelas parecería responder a un tipo de escuela artesanal de construcción colectiva, según estándares de calidad determinados y orientados a niveles secundarios de "excelencia». Entre otros aspectos, la participación de los padres es requerida para fomentar el desarrollo de la subjetividad y la personalidad. Entonces se conjugan los parámetros académicos con las necesidades individuales de cada alumno.

Las escuelas a la carta ofrecen nuevas opciones con relación al sector tradicional de oferta escolar y habilitan una diferenciación cultural y socioeconómica. Esta diferenciación se logra a partir de la oferta pedagógica y de la comunicación de sus ideales en sus páginas web. Las necesidades especiales que atienden responden a un alumnado con necesidades básicas satisfechas y que busca educarse a través del desarrollo de la subjetividad y la propia singularidad.

\subsection{Lógicas de ensamblaje: escuelas de largo plazo}

A diferencia de los centros analizados en el apartado anterior, hay un conjunto de escuelas en la Comuna 13 que ofrece los tres niveles de escolarización obligatoria según la legislación actualmente vigente. Estas escuelas, además de gozar de "prestigio", suelen ser instituciones «de moda», en las que es difícil ingresar, impedimento que se agudiza a medida que se asciende en los niveles educativos. Por un lado, porque la matrícula se completa casi en su totalidad desde el nivel inicial o primario y, por el otro, porque suelen ser escuelas bilingües que exigen un conocimiento de una segunda lengua muy avanzado para poder ingresar en ellas en el comienzo de otros niveles, en particular en el secundario. De esta manera, la propuesta institucional sugiere una matriculación temprana 
de los hijos en el nivel inicial, para luego «desligar» a los padres de tener que pensar - y repensar - futuras elecciones en los niveles subsiguientes. Así lo manifestó la directora de la escuela I: «Sí, claro, la idea es que los padres elijan un proyecto para los tres niveles y de alguna manera se olviden de tener que hacer futuras elecciones o decisiones sobre la trayectoria educativa de sus hijos».

Estas escuelas ofrecen una jornada extendida y, luego, actividades complementarias (talleres extracurriculares diversos, como fotografía, danzas, entre otros), de manera que los alumnos pueden pasar una gran parte de su tiempo allí, y es el lugar - físico y social— donde se desarrollan casi todas sus actividades, por lo que suelen posicionarse como un segundo hogar. Estas escuelas introducen programas complejos que apuntan a la preparación para la educación superior y formación de alumnos con "altas capacidades». Esto suma más dificultades para que ingresen nuevos alumnos en períodos tardíos, por un lado, y para que tanto padres como alumnos quieran cambiar de institución con nuevas etapas de la trayectoria educativa. Según la directora de la escuela L, "es muy difícil, porque, por una parte, no tenemos muchos chicos que se vayan [a otra secundaria]. Los padres que eligen esta escuela la eligen, si todo sale bien, para que se gradúen acá». En una línea de análisis similar, la psicopedagoga de la escuela K agrega: «Acá los grupos se mantienen estables. Son pocos los alumnos que no pueden seguir porque la escuela es muy exigente o porque tienen dificultades de aprendizaje».

Por otra parte, este tipo de escuelas apunta a la diferenciación por habilidades y también por valores. En particular, se trata de valores que refuerzan el sentido de comunidad, así como la preparación para la educación superior y la autonomía en el aula, con el objetivo de que se vea en el futuro reflejada en la autonomía profesional y laboral de los alumnos que se formaron en esa escuela. En esta línea de análisis y reafirmando el sentido de comunidad que crea la escuela, la directora de la escuela J afirmaba:

Es difícil que puedan ingresar nuevos alumnos con el nivel del idioma [se refiere a la segunda lengua que la escuela tiene y la vuelve bilingüe], aunque nosotros ofrecemos cursos de apoyo. Pero es difícil también porque se trata de un grupo ya armado desde primaria o jardín de infantes.

En estas instituciones, la pregunta no es si sus estudiantes seguirán estudiando — en niveles de educación universitaria—, sino qué y dónde, lo que también constituye un proyecto pedagógico en sí mismo, ya que se autoconciben como escuelas preuniversitarias. Esto último, y en línea con los hallazgos de una investigación reciente (Mayer, 2020), es intrínseco a las escuelas de élite $y$, agregamos aquí, a las escuelas que implementan lógicas de ensamblaje, en la medida en que se trata de un engranaje articulable a la trayectoria universitaria futura. Pero también se trata de un mecanismo que tiende a expulsar a alumnos que no pueden con las múltiples exigencias institucionales. Cuando las autoridades advierten estas situaciones, se les comunica a sus padres y, después de varias instancias, se los suele orientar respecto a otras posibilidades 
de escolarización. Así lo expresaba la directora de la escuela M: «Trabajamos mucho con los padres para que entiendan que tal vez esta escuela no es para sus hijos, que tiene un nivel de exigencia muy alto».

Esta mirada sobre la escuela guarda una estrecha relación con una mirada de exclusividad, anclada en estándares de "calidad» y "exigencia académica», que no parecen contemplar los ritmos personales de igual manera a las instituciones comprendidas en la lógica anterior. Sin embargo, resulta de interés destacar que las escuelas de ensamblaje también tienen ciertos rasgos comunes con las escuelas a la carta. Si bien se diferencian -y se excluyen mutuamente- en lo referido a ofrecer los tres niveles, plantean criterios basados en roles familiares y los docentes similares. Estas escuelas apelan de manera constante a los padres, ya que sin perder la "excelencia académica» se anclan fuertemente en vínculos personalizados (Van Zanten, 2009), con un despliegue de grandes niveles de protección y afecto: «Lo que intentamos hacer es generar hábitos en los chicos, que van de la mano de las virtudes, charlar con los chicos, conocerlos personalmente, y tratar que entre los docentes estén unidos» (directora de la escuela L).

Respecto a la valoración del desarrollo de la subjetividad, la personalidad y los recursos personales, estas instituciones basan su cultura en la creación de sentidos y pertenencia a una comunidad entendida como una segunda familia, por lo que los cambios de escuela al comenzar otro nivel o incluso dentro de un mismo nivel suponen la pérdida de un colectivo o tejido social donde acontece gran parte de la socialización del alumno y la familia.

\subsection{Lógicas vanguardistas: escuelas de punta}

Como toda gran metrópolis, la Ciudad de Buenos Aires encierra desigualdades que se expresan en su interior en el entramado urbano. Como destacan varios estudios (Mayer et al., 2020), en las últimas décadas, Buenos Aires, como la mayoría de las ciudades latinoamericanas, ha modificado su patrón de urbanización, caracterizado por procesos de reestructuración productiva y territorial, la financiarización de la ciudad y la fragmentación como lógica de producción del espacio (Dammert Guardia et al., 2019). A su vez, Buenos Aires experimentó un gran crecimiento frente a menores niveles de planificación urbana, lo que deriva en procesos de valorización creciente de la tierra y hace difícil para muchas escuelas adquirir terrenos amplios.

Dentro de este contexto, en la ciudad conviven escuelas creadas en casas antiguas que se fueron expandiendo con la compra de propiedades contiguas, pero que presentan escasos espacios para el esparcimiento y la movilidad. Estas escuelas realizan la actividad física en campos de deporte fuera de las instituciones, muchas veces en los márgenes de la ciudad, lo que supone una importante pérdida de tiempo en el traslado hacia el campo de deportes, por un lado, y la confirmación de la confinación y escasos espacios en el establecimiento, por el otro. El escenario opuesto se compone de un abanico de instituciones en la Comuna 13 que por ser de larga data pudieron conservar o adquirir grandes predios en los que alumnos y estudiantes pueden desarrollar múltiples activi- 
dades lúdicas y las correspondientes al currículo en materia de actividad física. Estas escuelas suelen privilegiar en sus diseños curriculares tanto la faceta lúdica como la deportiva como parte constitutiva de su identidad institucional.

Desde la perspectiva de análisis de los directores entrevistados, los padres pertenecientes a niveles socioeconómicos medios y medios-altos dicen valorar la infraestructura y el confort de sus hijos porque les permite desarrollar su personalidad y autonomía. «Hay padres que eligen la escuela por su oferta deportiva y su disponibilidad de espacios", afirmaba la directora de la escuela $\mathrm{K}$.

En este sentido, la infraestructura es entendida como el contexto escolar de aulas espaciosas, pero también como espacios al aire libre. El análisis de las entrevistas permitió ver que la infraestructura escolar es percibida como un dispositivo que o bien amplía o restringe la libertad individual y grupal, y también los modos en que los niños, niñas y jóvenes se relacionan entre sí y con las autoridades. El conjunto de elementos o amenities que la escuela ofrezca va a instaurar un clima institucional determinado y un ámbito de convivencia, y ambos van a operar como un dispositivo de selección de matrícula. En palabras de la directora de la escuela $\mathrm{H}$ :

En la página web no ponemos mucha información [respecto a la infraestructura] porque la gente ya conoce la escuela y los padres nunca preguntan, por ejemplo, si la escuela tiene pileta con agua climatizada porque lo dan por sentado, y quien lo pregunte es porque no conoce la escuela.

Asimismo, dentro de este grupo se encuentran las escuelas que, de manera más o menos concomitante a los grandes espacios, se anclan en la incorporación de las últimas tecnologías a las clases. En este marco, el proceso de matriculación incluye visitas guiadas para padres e hijos, y además se pudo observar que organizan reuniones especiales en horarios extraescolares donde concurren los docentes y los padres como parte de un dispositivo que facilita la comunicación acerca de la información que tienen los docentes sobre el desempeño de los alumnos.

En términos generales, los proyectos educativos de estas escuelas de punta se perciben como de vanguardia, con dimensiones extracurriculares que enriquecen la propuesta, tales como talleres de apoyo y refuerzo académico, participación en olimpíadas y programas de alumnos destacados, entre otros, de acuerdo con la información provista en la página web. El modelo pedagógico que se promueve es 2.0, se pone al alumno como centro del proceso de enseñanzaaprendizaje y se apunta a la formación en competencias. Según la información provista por los directores de las escuelas $\mathrm{H}$ y $\mathrm{K}$, los principios que orientan el proyecto pedagógico se basan en la "calidad en los resultados académicos», formación en "valores éticos», acompañamiento en el desarrollo académico, social, deportivo y afectivo de los alumnos, formación y capacitación docente, actualización constante de los planes de estudio, fomento de redes interescolares y colaboración con la comunidad. Esto hace, en opinión de las autoridades de estas instituciones, que su matrícula sea heterogénea, y más teniendo 
en cuenta la política de reducción arancelaria y becas, que varían desde un $20 \%$ hasta un $100 \%$ del arancel, con becas que incluso contemplan viático, vianda y ropa. La diferenciación que logran estas escuelas a través de su oferta basada en la incorporación de tecnología en las clases y estructuras edilicias que proyectan comodidad y esparcimiento hace que sean más atractivas para aquellos sectores de la sociedad que comparten una concepción educativa de vanguardia y privilegian la vida al aire libre y en contacto con la naturaleza, en el marco de sociedades urbanas donde esto último se torna cada vez más un recurso escaso y preciado.

\subsection{Lógicas globales: escuelas que se internacionalizan}

En línea con las últimas tendencias en reformas educativas que priorizan la incorporación de mecanismos de internacionalización, muchas escuelas - a partir de procesos y certificaciones diversos - han introducido proyectos que apuntan a desarrollar un mayor grado de conciencia respecto al cosmopolitismo y la apertura hacia el mundo. Los significados sobre qué es una escuela «abierta al mundo» son objeto de disputa, ya que para muchas instituciones el eje no está puesto en tal apertura, sino en los modos en que la misma se desarrolla (Larrondo y Mayer, 2018; Mayer, 2020). Aquí, nos interesa describir y analizar las lógicas de acción relacionadas con los modos en que las escuelas se internacionalizan tomando las escuelas binacionales, por un lado, y las escuelas que incorporan el Programa Diploma (PD) de la Organización del Bachillerato Internacional (IBO), por el otro.

Las escuelas binacionales son instituciones que se vinculan con dos sistemas educativos: el argentino, donde están radicadas y a cuyas normativas deben atenerse, y el sistema educativo de un segundo Estado nación, que en algunos casos financia parcialmente dichas escuelas y establece normas y diseños curriculares adicionales al local. Estas escuelas fueron creadas con el fin de asegurar procesos de inclusión diferenciados (Mayer y Schenquer, 2014; Mongiello, 2012), para descendientes de comunidades específicas que, fruto de los procesos y las olas de migración promovidos por el Estado argentino, se asentaron en el país. Esta relación centro-diáspora, fundante de estas instituciones, fue mutando a partir de los años ochenta y noventa, y la población «hijo de» o «nieto de» la comunidad de sentido que le da origen va declinando y comienza a conformase un público más heterogéneo en términos culturales y de procedencia geográfica y homogéneo en su aspecto socioeconómico.

Es importante resaltar que las escuelas que pertenecen a un sistema de un país determinado en el exterior conforman redes y relaciones entre ellas. Así, pertenecer por ejemplo a la Red de Escuelas Alemanas en el exterior habilita intercambios entre instituciones de diferentes países y no solo con las entidades del país referido. La directora de la escuela $\mathrm{N}$ afirmaba:

Nosotros, a través del programa [menciona un programa que amalgama diferentes instituciones relacionadas con un sistema educativo en el exterior], 
pudimos tomar contacto y hacer intercambios con escuelas de varios países, desde Brasil hasta escuelas europeas. Así, la escuela se nutre de alumnos de varios lugares.

Estos intercambios se hacen extensivos a los docentes, quienes a través de capacitaciones pedagógicas participan de eventos en el exterior. La directora de la escuela N agregaba: «El año pasado hubo una capacitación en el exterior de la que yo participé. Estuvo genial porque te relacionas con docentes de todo el mundo y te capacitas, capacitaciones que, si no, no tendría». Si bien en la actualidad las escuelas binacionales tienen fuertes vínculos —además del financiamiento- con la red de escuelas en el exterior del segundo Estado al que pertenecen, esta relación no está asociada a elementos centro-diaspóricos ni a la generación de procesos migratorios hacia esos países, sino a la utilización de ese vínculo como engranaje para garantizar una inclusión global de la escuela a través de viajes y movilidades educativas (Mayer y Catalano, 2018; Mayer, 2019) y presencias a nivel didáctico, pedagógico y de infraestructura (Larrondo y Mayer, 2018), entre otros aspectos que estarían garantizados a través de su filiación binacional.

Un segundo grupo de escuelas que se internacionaliza corresponde a las instituciones que se afilian a la Organización del Bachillerato Internacional (OBI), en particular a partir del Programa Diploma (PD) del Bachillerato Internacional. ${ }^{7}$ Este programa se ha instalado como un engranaje que intenta elevar el grado de atractividad y elegibilidad de la escuela. El programa se diferencia de otras plataformas por no ser un cúmulo de exámenes singulares que los estudiantes rinden, sino una certificación que, después de un arduo proceso de auditoría, les permite ser una escuela «del mundo IB», como suelen denominarse. Para las 60 escuelas que forman parte del mundo del Bachillerato Internacional en Argentina, el PD pasa a ser la columna vertebral de sus instituciones. La directora de la escuela L comentaba:

El Bachillerato Internacional te ordena, ordena la institución. Nosotros ya tenemos las fechas de examen del año que viene [2019] y del otro [2020], ya estamos preparando informes y otros documentos, algo que acá [en Argen-

7. La Organización del Bachillerato Internacional es una organización sin fines de lucro creada en 1968, con sede en Ginebra, para desarrollar programas de educación global, validados internacionalmente. En sus inicios, estos programas estuvieron pensados para las familias nómadas, esto es, para hijos de padres con profesiones con una gran movilidad laboral, pero luego se reformularon con los cambios sociales y las tendencias globalizantes en el mundo, lo que trajo aparejado la emergencia de los procesos de internacionalización de la educación (Mayer, 2019; 2020). Dentro de sus programas, destaca el Programa Diploma (PD), desarrollado para estudiantes de 16 a 18 años, que consiste en una plataforma específica de siete materias, el desarrollo de una investigación que culmina en un trabajo monográfico y actividades acreditables en aprendizaje y servicio solidario, el componente CAS del Programa. Aprobadas estas instancias tripartitas, los estudiantes acceden a una certificación que globaliza la validez de su título secundario y que permite el ingreso irrestricto a una gran parte de las universidades del mundo. 
tina] es imposible. Tenemos la planificación por los próximos cinco años. Y además no se puede pedir prórroga.

En similar línea de análisis, la directora de la escuela J afirmaba: «Ahora el proceso, la mirada, es diferente. $Y$ el proceso que está haciendo el colegio en ese sentido es ubicar el IB en ese lugar, como un eje inspirador, como un articulador de nuestra cultura institucional, algo que nos distingue de las demás escuelas".

A su vez, el PD actúa como un mecanismo para «ubicar la escuela en el mundo» (directora de la escuela $\mathrm{M}$ ), como consecuencia de las comparaciones posibles entre escuelas que pertenecen al «mundo IB» no solo en el país, sino en la región y en el mundo. La directora de la escuela $\mathrm{M}$ argumentaba:

El IB ayuda a la inserción, te obliga a tener una mirada más global de las cosas, porque todas las escuelas tienen el mismo programa. Te obliga pensar más en otro lado, a mirar más otro lado, se hacen más programas de intercambio, te van surgiendo más oportunidades, puedes compararte con escuelas en cualquier lugar del mundo.

Si bien el PD puede cursarse y rendirse en español, gran parte del universo que comprende a las escuelas del Bachillerato Internacional es «bilingüe». Son instituciones en las que el proceso de aprendizaje se realiza a través de dos lenguas o escuelas que destinan una gran carga horaria a segundos y terceros niveles que se certifican a través del PD. De este modo, las materias del programa se convierten en herramientas para fortalecer las habilidades bilingües de la institución. Tan es así que las escuelas binacionales suelen adherir el PD, muchas veces en conjunción con los exámenes de finalización del nivel secundario propio del segundo sistema educativo al que pertenecen. En esta primera dimensión, la adhesión al Bachillerato Internacional garantizaría un modelo de enriquecimiento con la segunda o la tercera lengua y de logros bilingües/trilingües. Sin embargo, los beneficios del programa, debido a su diseño curricular y más allá de la lengua en la que se dicte, son vistos como garante de una alfabetización internacional y multiculturalismo. La directora de la escuela $\mathrm{N}$ afirmaba:

[Con el PD] Ahí me parece que es interesante que el tipo de formación global que tiene que proveer una escuela de este tipo es una de integración a la globalidad a partir de la circulación de la particularidad, que es una de las formas de respetar la diversidad. Empezar a pensar y respetar la diversidad, en que soy diverso yo.

Tanto para las escuelas binacionales como para las pertenecientes al «mundo IB», los procesos y certificaciones que devienen de estas instancias son claves para garantizar el pasaje de escuelas bilingües a globales, donde estas instituciones intentan proveerse y proveer a sus estudiantes de un programa educativo amplio que los prepare para el mundo global (Banfi y Day, 2005; 
Mayer, 2020; Mongiello, 2012). En síntesis, el cosmopolitismo, o más precisamente la perspectiva internacional y global, tiene ramificaciones específicas y concretas en proyectos institucionales y diseños curriculares de las escuelas.

\section{Escuelas y posicionamiento social en cuasimonopolios educativos}

Las lógicas de acción de las escuelas y su consecuente posicionamiento social abren nuevas oportunidades para determinados sectores de la sociedad que pueden elegir la escuela para sus hijos. A su vez, es muy probable que la cercanía entre las escuelas de la Comuna 13 tienda a fomentar un mayor desarrollo de lógicas de acción y especificidad, de manera que el posicionamiento de una escuela sea la resultante de la relación de la diferenciación institucional marcada por su agencialidad, la respuesta a regulaciones estatales y las demandas de la comunidad educativa a las que pretende dar respuesta, entre otros aspectos. Estas nuevas identidades escolares ganan terreno a través de un triple proceso: a) las lógicas de acción implementadas por las escuelas, objeto de estudio de este artículo; b) las preferencias familiares de elección de escuela relevadas en una reciente publicación (Narodowski y Gottau, 2017; Gottau, 2020) y c) la ausencia de subvenciones estatales a las escuelas privadas (Tiramonti, 2011; Moschetti y Verger, 2020).

La contracara sería un mayor grado de fragmentación y segregación escolar frente a un Estado que abrió su función monopólica, en términos pedagógicos y educativos. El otrora Estado educador el siglo xx, al menos en los términos y condiciones en los que enraizó en la matriz educativa de Argentina, parecería estar cediendo lugar a nuevas identidades escolares. En este punto, resulta pertinente el análisis de Narodowski (2008), que sostiene que la actual configuración del sistema educativo argentino responde a la lógica de un «cuasimonopolio estatal». Este constructo teórico recupera el concepto de «cuasimonopolio", utilizado por Wallerstein (1984), y el de "crisis fiscal del Estado", de Aspremont y Gabszewicz (1985), para explicar que, en los casos en que el monopolio tradicional no logra satisfacer la demanda, surge como opción posible una oferta alternativa que puede actuar como bailout o "rescate». ${ }^{8}$ Dentro de los múltiples procesos que facilitan la emergencia de este sector, destacamos dos: (a) la instauración de mecanismos de financiamiento estatal a escuelas del subsistema privado de educación evidenciado en las subvenciones estatales que ofician como incentivo de «salida» (1970), ${ }^{9}$ y (b), en estrecha vinculación

8. Narodowski (2008) denomina «cuasimonopolio estatal» a la resultante del conjunto de la provisión del servicio educativo, que consta de un sector tradicional monopólico controlado por el Estado en términos de regulación, financiación y provisión, y un nuevo sector conformado por quienes están dispuestos a pagar por una educación que ya tienen pagada.

9. En términos de Hirschman (1970), podríamos argumentar que la opción de «salida» del sector estatal es una opción válida cada vez que la opción de "voz» resulta ineficaz o de un alto costo en cuanto a recursos, tiempo y esfuerzo invertido. En este sentido, para ciertos sectores de la sociedad, el mecanismo de «salida» es una opción menos costosa que el ejercicio de la «voz» en el sector público. Di John (2007) señala que un uso efectivo del mecanismo 
con nuestra investigación, la emergencia de circuitos educativos diferenciados mayormente ligados a sectores de «élite» como consecuencia de los procesos de masificación y extensión de la obligatoriedad escolar, donde ciertos públicos se repliegan. Estos procesos en paralelo no son completamente nuevos. En Argentina, la diversificación comienza a evidenciarse con más claridad a partir de 1960 (Braslavsky, 1985; Narodowski y Andrada, 2000) y se profundiza con los procesos de fragmentación propios de la década de los noventa, referentes a la pérdida de eficacia simbólica del Estado nación.

\section{Conclusión}

A lo largo de nuestro artículo, hemos analizado las lógicas de acción que llevan a cabo instituciones educativas de la Comuna 13 que no reciben financiamiento estatal dentro del marco conceptual de las lógicas de acción entendidas como lineamientos complementarios en muchos casos y no necesariamente excluyentes. Por lo tanto, resulta de interés resaltar nuevamente que una escuela puede adoptar múltiples lógicas de acción supeditando - o no- unas a otras, mientras que puede excluir otras. En particular, las lógicas que resultan excluyentes, desde un primer análisis, son las referidas a instituciones que ofrecen — o no- más de un nivel educativo.

Las escuelas que identificamos como a la carta o artesanales ponen énfasis en ser solo un eslabón en las trayectorias educativas, a diferencia de las propuestas que se orientan a completar - y contemplar - la totalidad de los niveles de escolarización obligatoria. Dentro de las múltiples explicaciones que manifiestan las autoridades de las escuelas a la carta para no extender su oferta al nivel subsiguiente —el secundario-, se destaca la idea de construir una «buena» $\mathrm{y}$ «exigente» escuela, pero con la contención a los tiempos individuales, que garantice $-\mathrm{O}$ al menos aumente- las probabilidades y posibilidades de ingresar a las escuelas de gestión estatal universitarias, caracterizadas por albergar públicos de élite intelectual al requerir altos puntajes en los exámenes de ingreso. Las escuelas cuyas lógicas son de ensamblaje o las correspondientes a las escuelas globales, como también lo marcamos en nuestro análisis, se presentan igualmente como instituciones preuniversitarias garantes de esos mismos niveles de «excelencia» que proponen tanto las primarias a la carta como las escuelas estatales dependientes de la universidad pública. Sin embargo, se orientan hacia otros repertorios relacionados con los procesos de internacionalización de la educación, la trasmisión de habilidades y saberes que suelen ser

de voz puede resultar más costoso que la salida «since [it] requires collective action, which is subject to the well-known difficulties of organization, representation and free-riding» (2007: 309). Sin embargo, por otro lado, también resulta de interés agregar que las subvenciones estatales a las escuelas privadas podrían no implicar una opción escolar o un incentivo para la elección o una estrategia de posicionamiento social para las escuelas. Ver JoHN, J. D. (2007). «Albert Hirschman's exit-voice framework and its relevance to problems of public education performance in Latin America». Oxford Development Studies, 35(3), 295-327. <https://doi.org/10.1080/13600810701514860> 
opacos en escuelas tanto estatales como privadas — por ejemplo, el dominio de una segunda $y$, muchas veces, una tercer lengua- y una construcción identitaria y comunitaria muy fuerte propia de instituciones que despliegan grandes estrategias para ser el centro de la trayectoria escolar $-\mathrm{y}$ vital- de sus alumnos y estudiantes.

Un punto intermedio son las escuelas agrupadas bajo la lógica de vanguardia, porque pueden o no ofrecer los tres niveles educativos, pero también porque su propuesta parece ser una interfaz entre las escuelas que privilegian lo individual, como las lógicas artesanales, y las comprendidas en las lógicas de ensamblaje o globales, por presentar mayores flexibilidades frente a las «exigencias» y heterogeneidades de logro académico, con la ponderación de la vida al aire libre, el desarrollo lúdico y el desarrollo motriz, capacidades que, si bien son valoradas, no se representan de igual manera que las derivadas del trabajo intelectual.

Siguiendo el análisis de Ball y Maroy (2009) respecto a las lógicas de acción, los autores destacan que a cada lógica le corresponde un «núcleo duro» o dimensión predominante, en torno a lo expresivo o instrumental. Los autores sostienen que mientras que las primeras ofrecen mayores niveles de apertura y heterogeneidad, las segundas proporcionan mayores niveles de homogeneidad en su público y, en consecuencia, de cierre social.

Entonces, las escuelas cuyas lógicas de acción se orientan a los procesos de internacionalización y al ensamblaje de la trayectoria educativa muestran una preeminencia de lo instrumental por encima de lo expresivo, ya que apuntan a una producción de saberes y conocimientos homogéneos y a estándares mínimos - pero muy altos al mismo tiempo-, mientras que las escuelas a la carta y las que se basan en su infraestructura y tecnología de punta presentan una prevalencia de lógicas expresivas a través del desarrollo de proyectos que destacan lo lúdico y recreativo, e intentan diferenciarse en torno a su infraestructura aunque sin descuidar la "excelencia» educativa. Sin embargo, puede suceder que existan casos que combinen lógicas de ensamblajes o globalizantes con las de tecnología de punta o infraestructura. Allí, las escuelas combinarían elementos a priori excluyentes, según la categorización de instrumental o expresiva. En estos casos, encontramos varios matices que giran en torno a dos extremos. En uno, una lógica queda supeditada a la otra, es decir, que, a modo de ejemplo, se privilegia lo internacional/global por encima de la infraestructura, de manera que lo que se identifica como prevalente es la instrumentalidad en grandes espacios o con tecnología de punta. En el otro extremo, encontramos instituciones donde estas combinaciones pueden ayudar a volver más «flexibles», en consecuencia, con mayores elementos expresivos, escuelas donde prima la instrumentalidad o, de manera inversa, aportar elementos instrumentales a núcleos expresivos que permiten «regular» los procesos formativos.

En conjunción con las lógicas de acción identificadas, el análisis hasta aquí expuesto permite comprender los procesos de diversificación de instituciones dentro de circuitos homologables entre sí. En línea con nuestro planteo inicial, este trabajo muestra que las escuelas no son actores pasivos o meros receptores 
de las elecciones de los padres. A través de sus lógicas de acción y estrategias de posicionamiento, intentan proyectar una imagen en línea con el proyecto institucional y el perfil de los alumnos o, más precisamente, el perfil de los padres y familias que desean albergar. Ahora bien, esta perspectiva respecto a la posibilidad de la aplicación de lógicas de acción diversa remite a cuestiones previas, basadas en anclajes socioeconómicos que desarrollaremos a continuación.

Estas escuelas generan espacios distintivos respecto al conjunto de escuelas que comprende tanto a las de gestión estatal como a las privadas con porcentajes de subvención que varían del $40 \%$ al $100 \%$. Este último porcentaje —o totalidad de la cuota - sucede en particular con las instituciones católicas.

La sociedad argentina, como toda organización social contemporánea, está vinculada a la globalización, lo que supone procesos de gran volatilidad, en particular para los países en vías de desarrollo o de desarrollo intermedio, que han visto reducidas sus capacidades de injerencia institucional como consecuencia de sus posiciones en la geopolítica mundial (Sidicaro, 2003; Mayer, 2009). De manera tal que, si las sociedades actuales se caracterizan por su liquidez e incertidumbre (Bauman, 2001; Beck Gernscheim, 2001), la argentina, tanto por procesos endógenos como exógenos, se presenta con mayores niveles de volatilidad (López y Romeo, 2005; Kessler, 2014; 2016). Sin embargo, una revisión por la estructura social del país desde la vuelta a la democracia (1983) muestra entre muchos factores niveles de estabilidad para los sectores mediosaltos y altos, los cuales, más allá de las vicisitudes y políticas socioeconómicas, logran conservar sus posiciones - y privilegios - en la pirámide local (Salvia y Piovani, 2018; Salvia y Cortés, 2019).

Como lo desarrollamos desde la introducción a nuestro artículo, las instituciones que analizamos se ubican en las zonas más privilegiadas de la ciudad, que es a su vez el enclave más rico del país, con indicadores favorables similares a las grandes metrópolis europeas (Mayer, 2020). Asimismo estas escuelas reclutan a los sectores más favorecidos de estos entramados, quienes en su mayoría pertenecen a las poblaciones que tienden a mantenerse por fuera de la coyuntura (Mayer et al., 2020). A diferencia de los sectores populares y de una gran proporción de los sectores medios y medios-bajos, que son los primeros en sufrir los impactos negativos de la política económica, estos no solo suelen permanecer con altos grados de impermeabilidad frente a los mismos, sino que los impactos acostumbran a ser positivos. Esto que puede vislumbrarse como un factor exógeno frente a la política educativa y a los proyectos institucionales está intensamente imbricado a ambos. El comportamiento educativo de la población argentina muestra, en términos generales, procesos migratorios del sector estatal al privado, que se agudizan cuanto más arriba nos encontramos en la pirámide social (Gamallo, 2015), de manera que el subsistema privado se torna cada vez más heterogéneo, al tiempo que recibe a diferentes poblaciones. Retomando nuestro planteo respecto a los sectores que reclutan estas escuelas, es importante volver a la estabilidad que estos presentan, ya que esta es condición sine qua non para el desarrollo de estas lógicas de acción presentadas. 
Para poder implementarse, ponerse en marcha y sostenerse, las lógicas de acción necesariamente requieren tiempo y recursos, tanto simbólicos como materiales. El sostenimiento de membresías — onerosas - con plataformas internacionales, la adquisición sistemática de tecnología de punta, el mantenimiento de grandes espacios o de lógicas de tipo boutique - que llevan a una relación de pocos alumnos por cada docente- son estrategias que para ser sostenibles solo pueden descansar en los privilegios de al menos amplios porcentajes de su base de reclutamiento. Dicho de otra manera, la sustentabilidad de estos proyectos está garantizada por las condiciones socioeconómicas arriba mencionadas.

En términos de política educativa, esto es relevante, ya que estas escuelas aparecen como producto de ambas dimensiones: los márgenes mayores de autonomía, por la ausencia de financiamiento económico estatal, y la posición socioeconómica de su público. Las instituciones que reciben financiamiento estatal, al tiempo que atienden a otros públicos dentro del sector privado, suelen reconocer que las que aquí analizamos - junto con otras de características similares pero que quedan por fuera de nuestro análisis por motivos diversos- son escuelas con las que no se pueden comparar ni pueden competir, lo que establece límites y fronteras. Esto a su vez justifica estas posiciones - y proyectos educativos - que se muestran excepcionales o inalcanzables, lo que colabora en la producción de nuevas desigualdades (Mayer, 2020). Entonces, podríamos argumentar que a diferencia de la discursividad escolar del siglo xx, que enfatizaba la igualdad y la homogeneidad, estas nuevas escuelas promueven un espacio diferente que se distingue del resto, y esta distinción aparece legitimada.

Así, este artículo realiza un importante aporte al analizar escuelas del subsistema privado sin subvención por parte del Estado. Si bien en este caso investigamos sus lógicas de acción y sus diversos grados de diversificación en el interior del sector privado, esto no implica que sea un fenómeno que solo atañe a este subsistema, ni que el sector estatal no establezca formas de diferenciación o diversificación interna. Futuras investigaciones podrían indagar si escuelas con una fuerte regulación estatal y con un menor manejo de los recursos logran desarrollar sus lógicas de acción.

\section{Referencias bibliográficas}

D'Aspremont Claude y Gabszewicz, Jean-Jaskold (1985). «Quasi-monopolies». Economica, 52 (206), 141-151. <https://doi.org/10.2307/2554416>

BALL, Stephen (1989). «Micro-politics versus management: Towards a sociology of school organization». Politics and the Processes of Schooling, 218-241.

Ball, Stephen J. y Maroy, Christian (2009). «School's logics of action as mediation and compromise between internal dynamics and external constraints and pressures». Compare, 39 (1), 99-112.

<https://doi.org/10.1080/03057920701825544> 
Banfi, Cristina y Day, Raymond (2005). «The evolution of bilingual schools in Argentina». En: Mejía, Anne-Marie de (eds.). Bilingual Education in South America. Clevedon.

Bauman, Zygmunt (2001). «Consuming life». Journal of Consumer Culture, 1 (1), 9-29.

Beck-Gernsheim, Elisabeth (2001). «Mujeres migrantes, trabajo doméstico y matrimonio. Las mujeres en un mundo en proceso de globalización». En: BECK-GERNSheim, E.; Butler, J. y Puigbert, L. Mujeres y transformaciones sociales. Barcelona: El Roure, 59-76.

BeEch, Jason y BARrenecheA, Ignacio (2011). "Pro-market educational governance: is Argentina a black swan?». Critical Studies in Education, 52 (3), 279-293. <https://doi.org/10.1080/17508487.2011.604077>

Bourdieu, Pierre (1987). «What makes a social class? On the theoretical and practical existence of groups». Berkeley Journal of Sociology, 32, 1-17.

- (2016). La distinction: critique sociale du jugement. Minuit.

Braslavsky, Cecilia (1985). La discriminación educativa en Argentina. Buenos Aires: FLACSO, Grupo Editor Latinoamericano.

Dammert Guardia, Manuel; Delgadillo, Víctor y Erazo, Jaime (2019). «Bibliografía sobre América Latina: nuevas desigualdades urbanas». Andamios, 16 (39), 255-262.

<https://doi.org/10.29092/uacm.v16i39.682>

Delvaux, Benard y Van Zanten, Agnes (2006). «Les établissements scolaires et leur espace local d'interdépendance». Revue Française de Pédagogie. Recherches en Éducation (156), 5-8.

<https://doi.org/10.4000/rfp.260>

John, J. D. (2007). «Albert Hirschman's exit-voice framework and its relevance to problems of public education performance in Latin America». Oxford Development Studies, 35(3), 295-327. <https://doi.org/10.1080/13600810701514860>

Gamallo, Gustavo (2015). «La "publificación” de las escuelas privadas en Argentina». Revista SAAP. Publicación de Ciencia Política de la Sociedad Argentina de Análisis Político, 9 (1), 43-74.

Gottau, Verónica (2020). «Private School Choice and Post-Materialism: What Values are at Stake?». Annual Review of Comparative and International Education 2019. Emerald Publishing Limited.

Hirschman, Albert (1970). Exit, voice, and loyalty: Responses to decline in firms, organizations, and states. Harvard University Press.

Kessler, Gabriel (2014). Controversias sobre la desigualdad. Buenos Aires: Fondo de Cultura Económica.

- (2016). Ideas y acciones en un contexto de cambio constante. Repensar la inclusión social. Buenos Aires: Capital Intelectual.

LARrondo, Marina y MaYer, Liliana (2018). Ciudadanías juveniles y educación: las otras desigualdades. Buenos Aires: Grupo Editor Universitario.

López, Artemio y Romeo, Martín (2005). La declinación de la clase media argentina. Transformaciones en la estructura social (1974-2004). Buenos Aires: Aurelia Rivera Grupo Editorial.

Maroy, Christian (2004). Regulation and inequalities in European education systems. Changes in regulation modes and social production of inequalities in education systems: A European comparison. Bruselas: Comisión Europea. 
Maroy, Christian y Van Zanten, Agnes (2009). «Regulation and competition among schools in six European localities». Sociologie du Travail, 51 (1), 67-79. <https://doi.org/10.1016/j.soctra.2009.01.005>

Mayer, Liliana (2009). Hijos de la democracia. ¿Cómo piensan y viven los jóvenes? Paidós.

- (2019). «Travelling to learn and learning by travelling. Educational Strategies at advantaged social Argentinian schools». Universitas, Revista de Ciencias Sociales y Humanas (30), 41-62.

- (2020). «Educación para todos y propuestas para pocos. La educación internacional en Argentina». En: Mayer, Liliana; Domínguez, María Isabel y Lerchundi, Mariana (eds.). Las desigualdades en clave generacional hoy: Las juventudes y las infancias en el escenario latinoamericano y caribeño. Buenos Aires: CLACSO.

Mayer, Liliana y Catalano, Bárbara (2018). "Internacionalización de la educación y movilidad: reflexiones a partir del caso argentino». Universitas, Revista de Ciencias Sociales y Humanas (29), 19-41 <https://doi.org/10.17163/uni.n29.2018.01>

Mayer, Liliana; Perozzo Ramírez, Wanda; Vázquez, Melina y Vommaro, Pablo (2020). «Desigual y diversa: producción de ciudad y vida urbana entre jóvenes de Buenos Aires». En: Mayer, Liliana; Duhalde, Juan Pablo; Arroyo Ortega, Adriana y Silva, María José (comps.). Ciudades x Jóvenes: Aportes a la Nueva Agenda Urbana de las Juventudes Latinoamericanas. Buenos Aires-Santiago de ChileBogotá: CLACSO- CINDE.

MAYer, Liliana y Schenquer, Laura (2014). «Europe outside Europe: developing a German Jewish citizenship in Argentina. The case of the Pestalozzi Schule». En: Galkowski, J \& Kotarski, H. Pragmatics of social and cultural capital. University of Rzeszow, Poland.

Mills, Wright (1956). The power elite. Londres: Oxford University Press.

Mongiello, Elena (2012). Cambios en la relación escuela y nación. Las escuelas italianas en el exterior: el caso argentino. Tesis de Maestría en Ciencias Sociales, FLACSO, Buenos Aires.

Morduchowicz, Alejandr (2005). «Private education: funding and (de) regulation in Argentina». Private Education and Public Policy in Latin America, 39.

Moschetti, Mauro Carlos y Verger, Antoni (2020). "Opting for private education: Public subsidy programs and school choice in disadvantaged contexts». Educational Policy, 34 (1), 65-90. <https://doi.org/10.1177/0895904819881151>

Narodowski, Mariano (2008). «School Choice and Quasi-State Monopoly in Education Systems in Latin America: the case of Argentina». En: Forsey, Martin; Davies, Scott y Walford, Geoffrey (eds.). The Globalisation of School Choice? Oxford: Symposium Books.

NARODOwsKI, Mariano y ANDrAdA, Myrian (2000). Segregación socioeconómica y regulaciones en el sistema educativo argentino. Buenos Aires: Centro de Estudios para el Desarrollo Institucional. Recuperado de <http://faculty.udesa.edu.ar/tommasi/cedi/dts/dt36.pdf>

Narodowski, Mariano y GotTau, Verónica (2017). «Clases medias y escuela pública. La elección escolar como resistencia». Perfiles Educativos, 39 (157), 34-51. <https://doi.org/10.22201/iisue.24486167e.2017.157.58439>

Narodowski, Mariano; Moschetti, Mauro Carlos y Gottau, Verónica (2017). «El crecimiento de la educación privada en Argentina: ocho explicaciones paradigmáticas». Cadernos de Pesquisa, 47 (164), 414-441.

<https://doi.org/10.1590/198053143853> 
Saltalamacchia, Homero (1992). La historia de vida: reflexiones a partir de una experiencia de investigación. Buenos Aires: Cijup.

Salvia, Agustín y Piovani, Juan Ignacio (2018). La Argentina en el siglo XXI: cómo somos, vivimos y convivimos en una sociedad desigual. Buenos Aires: Siglo XXI.

Salvia, Agustín y Cortés, Fernando (2019). Argentina y México. ¿Igualmente (des) iguales? Ciudad de México: Siglo XXI.

Sidicaro, Ricardo (2003). "Consideraciones sociológicas sobre la Argentina en la Segunda Modernidad». Estudios Sociales (24). <https://doi.org/10.14409/es.v24i1.2507>

Tiramonti, Guillermina (2004). La trama de la desigualdad educativa: mutaciones recientes en la escuela media. Buenos Aires: Ediciones Manantial.

- (2008). «As mutações da escola média na Argentina no marco da reconfiguração de um mundo globalizado». Pro-Posiçôes, 19 (3), 105-129. <https://doi.org/10.1590/s0103-73072008000300006>

- (2011). «La escuela media en su límite. Diferencias y continuidades en las configuraciones nacionales de la región: los casos de Brasil, Argentina y Chile. Différences et continuités des configurations nationales dans la région: les cas du Brésil, de l'Argentine et du Chili». Educação \& Sociedade, 32 (116), 857-875. <https://doi.org/10.1590/S0101-73302011000300013>

Torres, G. (2014). «Iglesia católica, educación y laicidad en la historia argentina». História da Educação, 18 (44), 165-185. <https://doi.org/10.1590/S2236-34592014000300010>

Van Zanten, Agnes (2009). "Competitive arenas and schools' logics of action: a European comparison». Compare, 39 (1), 85-98. <https://doi.org/10.1080/03057920802447867>

Verger, Antoni; Moschetti, Mauro y Fontdevila, Clara (2017). La privatización educativa en América Latina: una cartografía de politicas, tendencias y trayectorias. Barcelona: Universitat Autònoma de Barcelona.

Wallerstein, Immanuel (1984). The politics of the world-economy: The states, the movements and the civilizations. Cambridge University Press.

ZaNCAJO, Adrian (2018). «Educational providers in the marketplace: Schools' marketing responses in Chile». International Journal of Educational Research, 88, 166-176. <https://doi.org/10.1016/j.ijer.2017.10.009v> 


\section{Anexo}

Tabla 1. Descripción de la muestra intencional de escuelas primarias sin financiamiento estatal de la Comuna 13

\begin{tabular}{llllllll}
\hline Escuela & A & B & C & D & E & F & G \\
\hline Entrevistados & 2 & 2 & 2 & 2 & 2 & 2 & 2 \\
Orientación & Laica & Laica & Laica & Laica & Laica & Laica & Laica \\
Gestión & Privada & Privada & Privada & Privada & Privada & Privada & Privada \\
\% subvención estatal & $0 \%$ & $0 \%$ & $0 \%$ & $0 \%$ & $0 \%$ & $0 \%$ & $0 \%$ \\
IVS Comuna & Bajo & Bajo & Bajo & Bajo & Bajo & Bajo & Bajo \\
Comuna & 13 & 13 & 13 & 13 & 13 & 13 & 13 \\
\hline
\end{tabular}

Fuente: elaboración propia en base a la información provista por la Evaluación Integral de la Calidad y Equidad Educativa UEICEE (2019).

Tabla 2. Descripción de la muestra intencional de escuelas secundarias sin financiamiento estatal de la Comuna 13

\begin{tabular}{llllllll}
\hline Escuela & H & I & J & K & L & M & N \\
\hline Entrevistados & 2 & 2 & 2 & 2 & 2 & 2 & 2 \\
Orientación & Laica- & Laica- & Laica- & Laica- & Laica- & Laica & Laica \\
& técnica & bachiller comunidad italiana & comunidad judía & comunidad francesa \\
bilingüe & bilingüe \\
Gestión & Privada & Privada & Privada & Privada & Privada & Privada & Privada \\
$\%$ subvención estatal & $0 \%$ & $0 \%$ & $0 \%$ & $0 \%$ & $0 \%$ & $0 \%$ & $0 \%$ \\
IVS Comuna & Bajo & Bajo & Bajo & Bajo & Bajo & Bajo & Bajo \\
Comuna & 13 & 13 & 13 & 13 & 13 & 13 & 13 \\
\hline
\end{tabular}

Fuente: elaboración propia en base a la información provista por la Evaluación Integral de la Calidad y Equidad Educativa UEICEE (2019). 
